Departamento

de Enfermedades

Cardiovasculares, Escuela

de Medicina, Universidad de Valparaíso

${ }^{2}$ Departamento de

Cardiología y Cirugía

Cardiovascular, Clínica

Reñaca, Viña del Mar,

Chile.

Recibido el 2 de mayo de 2013, aceptado el 25 de octubre de 2013.

Los autores declararon no tener conflictos de intereses, utilizando e ICMJE Form for Disclosure

of Potential Conflicts of Interest.

Correspondencia a:

Dr. Rienzi Díaz Navarro Profesor Adjunto de Cardiología, Universidad de Valparaíso.

4 Poniente 332, Of. 305 ,

Viña del Mar, Chile.

Teléfono: 56-32-2658525

Fax: 56-32-2658527

E-mail: diaz.rienzi@gmail.

com

\section{Mixoma ventricular izquierdo asintomático diagnosticado circunstancialmente por ecocardiografía}

\author{
RIENZI DÍAZ ${ }^{1,2}$, ERNESTO ARÁNGUIZ ${ }^{1,2}$
}

We report a 50 year-old hypertensive and asymptomatic woman in whom a left ventricular tumor was found on a routine two-dimensional transthoracic echocardiogram. Complementary 3D echocardiography, cardiac magnetic resonance and cardiac computed tomography allowed the diagnosis of a calcified left ventricular myxoma. Surgical treatment was performed through a transaortic transvalvular approach with tumor resection and implantation of a pericardial bovine patch. This case confirms the importance of transthoracic echocardiography in the diagnosis of cardiac tumors and the complementary role of $3 D$ echocardiography, cardiac magnetic resonance imaging and computed tomography for the differential diagnosis before surgery.

(Rev Med Chile 2013; 141: 1594-1597)

Key words: Echocardiography; Heart neoplasms; Myxoma.

$\mathrm{E}$ 1 mixoma es el más común de los tumores cardiacos y representa aproximadamente a $50 \%$ de las neoplasias primarias. La mayoría se localiza en la aurícula izquierda (97-98\%) y sólo 2-3\% en los ventrículos ${ }^{1,2}$. La sintomatología depende de su localización, aunque ocasionalmente no producen síntomas y su pesquisa es posible por el uso cada vez más frecuente de la ecocardiografía ${ }^{3,4}$.

\section{Caso clínico}

Mujer de 50 años, hipertensa, asintomática. En control ambulatorio rutinario se solicitó un ecocardiograma bidimensional transtorácico (Eco2D), luego de la auscultación de un soplo sistólico III/VI en el foco aórtico. El Eco2D reveló la existencia de una masa móvil al interior del ventrículo izquierdo de 5,0 x 1,3 cm, adherida al septum interventricular, con zonas de intensidad aumentada, sugerentes de calcificación (Figura 1, panel A), que provocaba una obstrucción parcial del tracto de salida del ventrículo izquierdo (TSVI), con gradiente máxima de $26 \mathrm{mmHg}$ (Figura 1, panel B). No se observaron tumores adicionales en ninguna otra cámara cardiaca y los exámenes de laboratorio, radiografía de tórax y electrocardiograma resultaron normales. Para una mejor evaluación de la masa intraventricular, se realizó ecocardiografía tridimensional (Eco 3D), que permitió identificar la morfología multilobular del tumor, objetivándose -además- que ocupaba 65\% del TSVI (Figura 1, panel C y D). Para complementar la evaluación, se efectuó resonancia cardiaca (RC) que mostró un tumor con señal isointensa respecto del tejido miocárdico y un pedúnculo de anclaje de $2 \mathrm{~cm}$ adherido al tercio superior del septum interventricular superior, que no interfería con el normal funcionamiento del aparato subvalvular mitral (Figura 1, panel E). En la imagen de inversión/recuperación posterior 

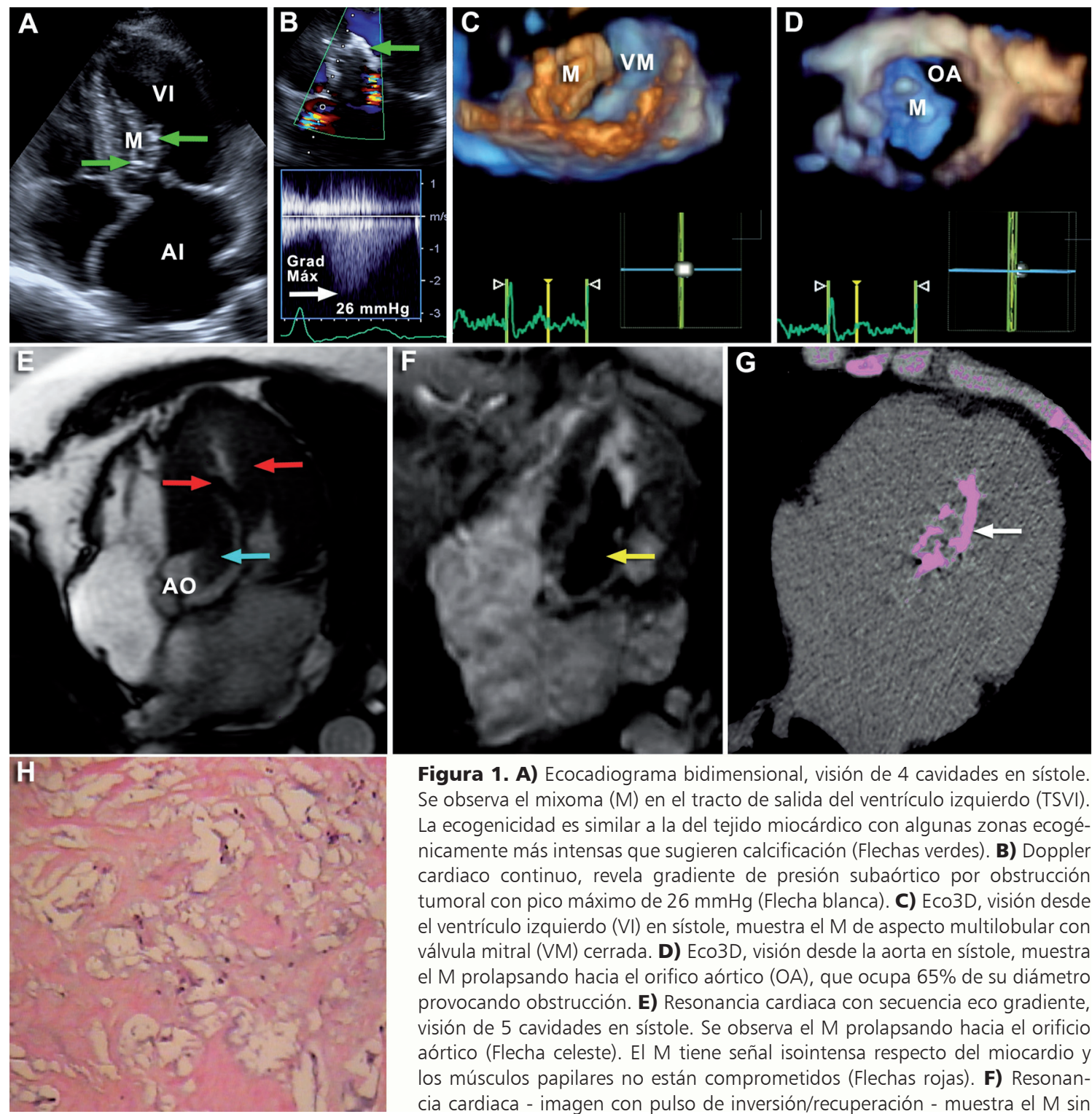

Figura 1. A) Ecocadiograma bidimensional, visión de 4 cavidades en sístole. Se observa el mixoma (M) en el tracto de salida del ventrículo izquierdo (TSVI). La ecogenicidad es similar a la del tejido miocárdico con algunas zonas ecogénicamente más intensas que sugieren calcificación (Flechas verdes). B) Doppler cardiaco continuo, revela gradiente de presión subaórtico por obstrucción tumoral con pico máximo de $26 \mathrm{mmHg}$ (Flecha blanca). C) Eco3D, visión desde el ventrículo izquierdo $(\mathrm{VI})$ en sístole, muestra el $\mathrm{M}$ de aspecto multilobular con válvula mitral (VM) cerrada. D) Eco3D, visión desde la aorta en sístole, muestra el M prolapsando hacia el orifico aórtico (OA), que ocupa $65 \%$ de su diámetro provocando obstrucción. E) Resonancia cardiaca con secuencia eco gradiente, visión de 5 cavidades en sístole. Se observa el M prolapsando hacia el orificio aórtico (Flecha celeste). El M tiene señal isointensa respecto del miocardio y los músculos papilares no están comprometidos (Flechas rojas). F) Resonancia cardiaca - imagen con pulso de inversión/recuperación - muestra el M sin realce tardío muy probablemente por la importante calcificación del tumor (Flecha amarilla). G) TCMC para estudio de calcio muestra intensa calcificación del M codificada en color rosa (Flechas blancas). H) Estudio histológico del mixoma con tinción tricrómica de van Gieson muestra células poligonales inmersas en matriz de mucopolisacáridos que caracterizan este tumor. TCMC, tomografía computada multicorte de corazón.

a la administración de gadolinio, no se observó contraste espontáneo en el tumor (Figura 1, panel F). Por último, la evaluación del paciente se complementó con tomografía computada multicorte de corazón (TCMC), que permitió comprobar la existencia de zonas tumorales calcificadas sospechadas en el Eco2D (Figura 1, panel G). Con el diagnóstico de mixoma de ventrículo izquierdo, la paciente fue operada realizándose la resección quirúrgica del tumor vía transvalvular aórtica, que incluyó su base de anclaje y la implantación posterior de un parche de pericardio bovino. La válvula mitral y su aparato subvalvular eran normales. El análisis histopatológico del tumor comprobó el diagnóstico de mixoma calcificado (Figura 1, panel $\mathrm{H}$ ). 


\section{Discusión}

El mixoma cardiaco es el tumor primario de corazón más frecuente, con una prevalencia de hasta $50 \%$ en estudios necrópsicos, la mayoría con localización en las aurículas ${ }^{1,2}$.

Los mixomas son tumores histológicamente benignos, cuya sintomatología depende de su localización. Sin embargo, hay tumores de tamaño significativo -como el de nuestra paciente- que pueden no producir síntomas ${ }^{3,5}$. Los fenómenos embólicos, el síncope y los soplos sistólicos por obstrucción del TSVI, son las manifestaciones clínicas más frecuentes del mixoma ventricular izquierdo ${ }^{6}$, aunque muy ocasionalmente puede asociarse a arritmias cardiacas ${ }^{7}$. Pero, la complicación más frecuente es la embolia sistémica que puede incluso provocar obstrucción de la aorta abdominal y arterias renales ${ }^{8,9}$.

El mixoma ventricular izquierdo en nuestra paciente se descubrió de manera incidental mediante Eco2D, solicitado para la evaluación de un soplo sistólico pesquisado en un control rutinario de su hipertensión arterial.

Antes del año 1951, el diagnóstico de los tumores intracardiacos se hacía exclusivamente en estudios necrópsicos ${ }^{6}$. En 1952, fue posible diagnosticar en vida un mixoma de aurícula izquierda mediante angiografía ${ }^{10}$. Posteriormente, la incorporación de la ecocardiografía a la práctica clínica permitió diagnosticar por primera vez un mixoma de la aurícula izquierda mediante ecocardiografía modo $\mathrm{M}^{11}$. Desde entonces, este método de diagnóstico es relevante para el diagnóstico del mixoma cardiaco, haciendo posible su identificación preoperatoria en aproximadamente $90 \%$ de los $\operatorname{casos}^{12}$. Por otra parte, la Eco3D permite actualmente estimar con mayor exactitud la morfología tumoral, el tamaño del tumor y el grado de obstrucción que ocasiona al flujo sanguíneo. En nuestra paciente, la Eco3D reveló el aspecto multilobular del mixoma y también demostró que ocupaba $65 \%$ del orificio aórtico, provocando obstrucción del TSVI (Figura 1, panel C y D).

Si bien la ecocardiografía en sus distintas modalidades es la técnica más usada para la pesquisa y el diagnóstico de los tumores cardiacos, actualmente existen otros métodos de diagnóstico no invasivo -entre los que destaca la RC- que entregan información relevante de la anatomía del tumor, composición, vascularización y su relación con los tejidos vecinos, información sustantiva en la evaluación preoperatoria de estos pacientes. En la actualidad, la RC es considerada una indicación Clase I en la evaluación de los tumores cardiacos ${ }^{13}$.

La RC en nuestra paciente confirmó el grado de obstrucción del TSVI observado en la Eco3D y permitió determinar con exactitud la extensión de la base de anclaje del mixoma en la porción superior del septum interventricular en una extensión de $2 \mathrm{~cm}$, información preoperatoria de gran importancia. Por último, la sospecha de calcificación del tumor por Eco2D fue confirmada por TCMC, hallazgo que constituye una característica histopatológica en algunos mixomas.

La información obtenida con el uso de los distintos métodos de diagnóstico permitió realizar el diagnóstico de un mixoma de ventrículo izquierdo calcificado que fue comprobado en el análisis histopatológico del tumor extirpado (Figura 1, panel H). Sin embargo, es importante advertir que, para definir una adecuada conducta terapéutica en un tumor cardiaco -como el de nuestro paciente- bastaría con la ecocardiografía inicial complementada con el estudio de resonancia cardiaca.

El diagnóstico de mixoma de ventrículo izquierdo requiere cirugía de urgencia por el alto índice de eventos embólicos que aumentan la mortalidad de estos pacientes ${ }^{14}$.

Para la cirugía de un mixoma ventricular izquierdo, es crucial poseer información preoperatoria segura, especialmente del tamaño, forma, movilidad, textura y sitio de anclaje tumoral, para definir el procedimiento quirúrgico más apropiado. En este sentido, la RC es fundamental para determinar la extensión de la lesión en el miocardio. En el caso de nuestra paciente, la vía de abordaje fue transáortica, porque el mixoma estaba localizado en el TSVI, mientras que para otras localizaciones la vía utilizada es la auricular. La resección del mixoma no requiere de la extirpación completa de la pared ventricular, porque no se han observado recidivas cuando se realizan resecciones más limitadas ${ }^{15}$.

En síntesis, una paciente portadora de un mixoma ventricular izquierdo con obstrucción parcial del TSVI sin sintomatología asociada, incidentalmente diagnosticado por Eco2D. El diagnóstico diferencial preoperatorio fue posible con el uso combinado de Eco3D, RC y TCMC. 


\section{Referencias}

1. Roberts WC. Primary and secondary neoplasms of the heart. Am J Cardiol 1997; 80: 671-82.

2. Becker O, Ramírez A, Zalaquett S, Morán S, Irarrázaval MJ, Arretz S, et al. Mixoma cardiaco: Caracterización clínica, métodos diagnósticos y resultados alejados del tratamiento quirúrgico durante tres décadas de experiencia. Rev Med Chile 2008; 136: 287-95.

3. Bicer A, Turhan H, Cagrici G, Yasar AS, Sasmaz H. Asymptomatic left ventricular myxoma diagnosed incidentally by transthoracic echocardiography. Echocardiography 2005; 22: 855-6.

4. Keeling IM, Oberwalder P, Anelli-Monti M, Schuchlenz H, Demel U, Tilz GP, et al. Cardiac myxomas: 24 years of experience in 49 patients. Eur J Cardiothorac Surg 2002; 22 (6): 971-7.

5. Lamparter S, Moosdorf R, Maisch B. Giant Left atrial mass in an asymptomatic patient. Heart 2004; 90 (5): e24.

6. Reynen K. Cardiac Myxomas. Review article. N Engl J Med 1995; 333: 1610-7.

7. Bauer MF, Aebert H, Zurbrugg H, Rushoff J, Birnbaum DE. Torsade de pointes arrhythmia in a patient with left ventricular myxoma. Chest 1994; 105: 1876-8.

8. Young RD, Hunter WC. Primary myxoma of the left ventricle with embolic occlusion of the abdominal aorta and renal arteries. Arch Pathol 1947; 43: 86-91.

9. Kay JH, Anderson RM, Meihaus J, Lewis R, Magidson O, et al. Surgical Removal of an Intracavitary Left Ventricular Myxoma. Circulation. 1959; 20: 881-6.

10. Golberg HP, Glenn F, Dotter CT, Steinberg I. Myxoma of the left atrium: diagnosis, made during life with operative and postmortem findings. Circulation 1952; 6: 762-7.

11. Pavlides GS, Levin RN, Hauser AM. Left ventricular recurrence of a resected left atrial myxoma. Am Heart J 1989; 117 (6): 1390-2.

12. Sugeng L, Lang RM. Atypical cardiac myxomas. Echocardiography 2004; 21 (1): 43-7.

13. Penell DJ, Sechtem UP, Higgins CB, Manning WJ, Pohost GM, Rademakers FE, et al. Clinical indications for cardiovascular magnetic resonance (CMR): Consensus Panel Report. Eur Heart J 2004; 25: 1940-65.

14. Thongcharoen P, Laksanabungsong P, Thongtang V. Left ventricular outflow tract obstruction due to a left ventricular myxoma: a case report review of the literature. J Med Assoc Thai 1997; 80: 799-806.

15. Bartolotti U, Maraglino G, Rubino M, Santini F, Mazzucco A, Milano A, et al. Surgical excision of intracardiac myxomas: a 20-year follow-up. Ann Thorac Surg 1990; 8: 673-82. 